\title{
Magnetic screening properties of superconductor-ferromagnet bilayers
}

\author{
Manuel Houzet ${ }^{1}$ and Julia S. Meyer ${ }^{2,3}$ \\ ${ }^{1}$ INAC, SPSMS, CEA, F-38054 Grenoble, France \\ ${ }^{2}$ Department of Physics, The Ohio State University, Columbus, Ohio 43210, USA \\ ${ }^{3}$ Université Joseph Fourier, F-38041 Grenoble, France
}

(Received 12 March 2009; revised manuscript received 18 June 2009; published 14 July 2009)

\begin{abstract}
We study theoretically the magnetic screening properties of thin, diffusive superconductor/ferromagnet bilayers subject to a perpendicular magnetic field. We find that the effective penetration depth characterizing the magnetic response oscillates with the thickness of the ferromagnetic layer on the scale of the ferromagnetic coherence length.
\end{abstract}

DOI: 10.1103/PhysRevB.80.012505

PACS number(s): 74.78.Fk, 74.25.Ha, 74.25.Nf, 74.45.+c

While superconductor-normal metal (SN) structures have been intensively studied for decades, superconductorferromagnet (SF) structures have only become accessible recently because of the much-reduced length scales in ferromagnets. Due to their incompatible spin properties, the proximity effect between a singlet superconductor and a ferromagnet leads to a variety of unusual phenomena. ${ }^{1,2}$ Through the exchange field acting on the electron spins in the ferromagnet, Cooper pairs acquire a finite-momentum $\delta k$ which leads to an oscillatory behavior of the anomalous Green's function. ${ }^{3}$ Observable consequences are, e.g., a nonmonotonic dependence of the transition temperature ${ }^{4-6}$ and the density of states at the Fermi level ${ }^{7,8}$ on the thickness of the F layer in SF bilayers, and the possibility of $\pi$-Josephson junctions at certain thicknesses of the $F$ layer in superconductor-ferromagnet-superconductor trilayers. $^{9-11}$

(SFS)

While most experiments on hybrid systems use resistive measurements, screening of an external magnetic field offers an alternative tool to study the proximity effect. These measurements probe deeply into the superconducting state because they provide both the magnitude and temperature dependence of the effective superfluid density. Various configurations for the magnetic response can be considered. The magnetization of SN hybrids with a magnetic field applied parallel to their interface has been addressed theoretically in Ref. 12, with still debated experimental results in the case of SN cylinders. ${ }^{13,14}$ Alternatively, the screening properties of thin films can be probed by measuring the mutual inductance of two coils positioned on opposite sides of the sample..$^{15,16}$ The mutual inductance can be related to the complex conductivity of the film which in turn can be related to the screening-length $\lambda$ or the superfluid-density $\rho_{S}$. To be precise, in SF bilayers, one measures the superfluid-density $\rho_{S} \propto \lambda^{-2}$ integrated over the width of the bilayer or an effective screening-length

$$
\lambda_{\mathrm{eff}}^{-2}=d_{S}^{-1} \int_{-d_{S}}^{d_{F}} d x \lambda^{-2}(x),
$$

where $d_{S}$ and $d_{F}$ are the thicknesses of the superconducting and ferromagnetic layer, respectively, and $x$ is the coordinate normal to the interface. First experimental results on $\mathrm{Nb} / \mathrm{Ni}$ bilayers have been reported using this setup in Ref. 17, where a nonmonotonic dependence of the effective screening-length on the thickness of the Ni layer has been observed.

In this Brief Report, we study the screening-length $\lambda_{\text {eff }}$ of a SF bilayer subject to a weak perpendicular magnetic field. The main assumptions are that $(i)$ the exchange-field $h$ in the ferromagnet is much larger than the superconducting orderparameter $\Delta$, (ii) the system is in the dirty limit and, thus, the Usadel equation ${ }^{18}$ can be used, (iii) the screening-length $\lambda_{\text {eff }}$ is much larger than the thickness $d=d_{S}+d_{F}$ of the bilayer, and (iv) the width $d_{S}$ of the superconducting (S) layer is smaller than the superconducting coherence-length $\xi_{S}$ $=\sqrt{D_{S} /\left(2 \pi T_{c 0}\right)}$, where $D_{S}$ is the diffusion constant and $T_{c 0}$ is the transition temperature of the bare $\mathrm{S}$ layer. Our main result is that the screening length displays an oscillatory behavior with the thickness of the ferromagnet.

Due to the normalization-condition $\hat{g}^{2}=1$ of the quasiclassical Usadel Green's-function $\hat{g}$, it can be parametrized by an angle $\theta$ such that the normal Green's-function $G=\cos \theta$ whereas the anomalous Green's-function $F=\sin \theta$. The system is then described by four coupled equations in terms of the angles $\theta_{S}$ on the $S$ side of the SF interface, $\theta_{0}$ on the ferromagnetic $(\mathrm{F})$ side of the SF interface, and $\theta_{F}$ at the ferromagnet-vacuum interface.

The Usadel equation of the $\mathrm{F}$ layer, $-D_{F} \nabla^{2} \theta+2 i h \sin \theta$ $=0$, can be integrated to yield

$$
2 \sqrt{i y}=\int_{\theta_{F}}^{\theta_{0}} d \theta \frac{1}{\sqrt{\cos \theta_{F}-\cos \theta}},
$$

where $y=d_{F} / \xi_{F}$ and $\xi_{F}=\sqrt{D_{F} / h}$ is the ferromagnetic coherence length, with the diffusion-constant $D_{F}$ of the $\mathrm{F}$ layer. The boundary condition imposing current conservation at the $\mathrm{SF}$ interface ${ }^{19}$ can be expressed as

$$
\sin \left(\theta_{S}-\theta_{0}\right)=2 \sqrt{i} \beta \sqrt{\cos \theta_{F}-\cos \theta_{0}},
$$

where $\beta=R_{b} \sigma_{F} / \xi_{F}$. Here $R_{b}$ is the interface resistance per square, and $\sigma_{F}$ is the conductivity of the $\mathrm{F}$ layer. In the limit $d_{S} \ll \xi_{S}$, the Usadel equation of the $\mathrm{S}$ layer, $-D_{S} \nabla^{2} \theta$ $+2 \omega \sin \theta=2 \Delta \cos \theta$, where $\omega$ is a fermionic Matsubara frequency, can be simplified by an expansion in small spatial variations of the angle $\theta$ across the S layer combined with the boundary condition (3). One obtains 


$$
\omega \sin \theta_{S}+2 \sqrt{i \alpha} \sqrt{\cos \theta_{F}-\cos \theta_{0}}=\Delta \cos \theta_{S},
$$

where $\alpha=D_{S} \sigma_{F} /\left(2 \sigma_{S} d_{S} \xi_{F}\right)$ and $\sigma_{S}$ is the conductivity of the $\mathrm{S}$ layer. Finally, the self-consistency equation for the orderparameter $\Delta$ reads

$$
\Delta=\pi T \lambda_{\mathrm{BCS}} \Re\left[\sum_{\omega} \sin \theta_{S}\right]
$$

where $\lambda_{\mathrm{BCS}}$ is the Bardeen-Cooper-Schrieffer coupling constant.

In diffusive superconductors, the screening length $\lambda$ describes the local (London) current response ${ }^{20}$ to a vector potential, $\boldsymbol{j}=-1 /\left(\mu_{0} \lambda^{2}\right) \boldsymbol{A}$, where $\lambda^{-2}=\left(2 \pi T \mu_{0} \sigma_{S} / \hbar\right) \sum_{\omega} F^{2}$ is proportional to the superfluid density. Here $\mu_{0}$ is the vacuum permeability. In SF bilayers, the effective screening length is related to the angles $\theta$ through the equation

$$
\frac{1}{\lambda_{\mathrm{eff}}^{2}}=\frac{2 \pi T \mu_{0} \sigma_{S}}{\hbar} \mathfrak{R}\left[\sum_{\omega}\left(\sin ^{2} \theta_{S}+\gamma \int_{0}^{y} d x \sin ^{2} \theta(x)\right)\right],
$$

where $\gamma=\sigma_{F} \xi_{F} /\left(\sigma_{S} d_{S}\right)$. Using the Usadel equation of the $\mathrm{F}$ layer, the integral over $x$ can be traded for an integral over $\theta$, namely $d x=\frac{1}{2 \sqrt{i}}\left(\cos \theta_{F}-\cos \theta\right)^{-1 / 2} d \theta$, ranging from $\theta_{F}$ to $\theta_{0}$.

In general the set of Eqs. (2)-(5) can be solved numerically only, but in some limiting cases an analytical solution is possible. At $T=0$, a simplification occurs because the sums over $\omega$ can be replaced by integrals, and subsequently the integration over $\omega$ can be traded for an integration over $\theta_{S}$ using the Usadel equation. ${ }^{21}$ It is then sufficient to solve the Usadel equation at $\omega=0$ for $\theta_{S}(0)$. In particular, using Eqs. (2) and (3), the Usadel equation of the $S$ layer (4) can be brought into the form $\left[\omega+F\left(\theta_{S}\right)\right] \sin \theta_{S}=\Delta \cos \theta_{S}$, yielding $d \omega=-\left(\Delta / \sin ^{2} \theta_{S}+F^{\prime}\left(\theta_{S}\right)\right) d \theta_{S}$. Using this trick, the zerotemperature gap $\Delta$ is given as

$$
\ln \frac{\Delta}{\Delta_{0}}=\Re\left[\ln \tan \frac{\theta_{S}(0)}{2}+\int_{0}^{\theta_{S}(0)} d \theta_{S} F^{\prime}\left(\theta_{S}\right) \sin \theta_{S}\right],
$$

where $\Delta_{0}$ is the zero-temperature gap of the bare S layer, a result which can then be used to compute $\lambda_{\text {eff- }}^{-2}$.

In the following, we provide analytical results for the effective screening length in two limits, namely $(i)$ for a system without barrier $\beta=0$ and (ii) for a system with a strong barrier $\beta \gg 1$. In both cases, solutions are presented for small parameters $\alpha$. For convenience, we introduce the notation $\tilde{x}$ $=(1+i) x$ for $x=\alpha, \beta, y$.

In the absence of a barrier $\beta=0$, the boundary condition (3) imposes that the angles on both sides of the SF interface, $\theta_{0}$ and $\theta_{S}$, are the same.

If $d_{F} \gg \xi_{F}$, the angle $\theta_{F}$ is small, and Eq. (2) yields $\theta_{F}$ $=8 \tan \frac{\theta_{S}}{4} \exp [-\tilde{y}]$. Thus, we can simplify Eq. (4) to yield

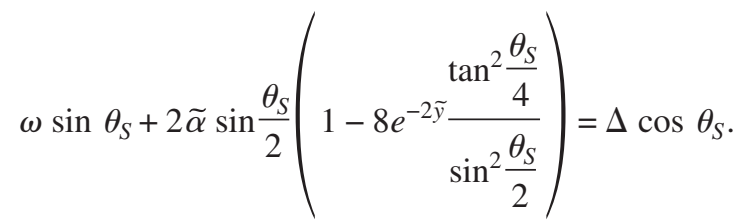

Treating $\alpha \ll \Delta_{0}$ perturbatively, one finds $\theta_{S}(0)=\frac{\pi}{2}+\delta \theta_{S}$, where $\delta \theta_{S}=-\sqrt{2}\left(\tilde{\alpha} / \Delta_{0}\right)\left[1-16 e^{-2 \tilde{y}}(3-2 \sqrt{2})\right]$, and

$$
\begin{aligned}
\delta \Delta= & -2 \alpha[\sqrt{2}-\ln (1+\sqrt{2})] \\
& -\frac{16}{3} \Re\left[\tilde{\alpha} e^{-2 \tilde{y}}\right][3 \ln (1+\sqrt{2})+4-5 \sqrt{2}] .
\end{aligned}
$$

This solution describes gapless superconductivity with a finite density-of-states $\nu(0)$ at the Fermi level in the superconductor that oscillates with the thickness of the ferromagnet: $\nu(0)=-\nu_{0} \Re\left[\delta \theta_{S}\right]$, where $\nu_{0}$ is the density of states in normal state. The equation for the screening length takes the form

$$
\begin{aligned}
\frac{\lambda_{0}^{2}}{\lambda_{\mathrm{eff}}^{2}(0)}= & 1-\left(a_{1}-a_{2}(\cos 2 y+\sin 2 y) e^{-2 y}\right) \frac{\alpha}{\Delta_{0}} \\
& +\frac{2 \sqrt{2}}{3 \pi} \gamma\left(1+a_{3} y e^{-2 y} \cos 2 y-a_{4} \frac{\alpha}{\Delta_{0}}\right),
\end{aligned}
$$

where $\lambda_{0}^{-2}=\pi \mu_{0} \sigma_{S} \Delta_{0} / \hbar$ is the inverse screening length of the bare $S$ layer at zero temperature, and the coefficients $a_{i}$ are positive. $^{22}$ Both the contributions to the effective screening length from the S layer and from the F layer $(\propto \gamma)$ oscillate on the length scale of the ferromagnetic coherence length.

If, on the other hand $d_{F} \ll \xi_{F}$, the variation of the angle $\theta$ is small across the $\mathrm{F}$ layer and, thus, $\theta_{S}-\theta_{F} \ll 1$. Using Eq. (2), one obtains $\theta_{S}=\theta_{F} \cosh \tilde{y}$. Inserting this relation into the Usadel equation of the $\mathrm{S}$ layer results in

$$
\left(\omega+2 i \alpha y+\frac{4}{3} \alpha y^{3} \cos \theta_{S}\right) \sin \theta_{S}=\Delta \cos \theta_{S} .
$$

We find $\delta \theta_{S}=-2 i \alpha y / \Delta_{0}$ and $\delta \Delta=-\frac{\pi}{3} \alpha y^{3}$. Note that because $\theta_{S}(0)=\frac{\pi}{2}+i \phi$, where $\phi$ real, the density of states possesses a gap in this regime. Using Eq. (11) to convert the integral over $\omega$ to an integral over $\theta_{S}$, the screening length is given by

$$
\frac{\lambda_{0}^{2}}{\lambda_{\mathrm{eff}}^{2}(0)}=1-\frac{\pi}{3}\left(1+\frac{16}{3 \pi^{2}}\right) \frac{\alpha}{\Delta_{0}} y^{3}+\gamma y \text {. }
$$

Equation (12) predicts an increase in $\lambda_{\text {eff }}^{-2}$ as long as $d_{F}$ $<\xi_{F}^{2} / \xi_{S}$ before it starts to decrease. The regime $d_{F} \sim \xi_{F}$ connecting the results Eqs. (10) and (12) is treated numerically (see below).

In the opposite limit of a strong barrier, $\beta \gg 1$, both $\theta_{0}$ and $\theta_{F}$ are small, if the $\mathrm{F}$ layer is not too thin, $y \gg \beta^{-1}$. Equation (2) then yields $\theta_{0}=\theta_{F} \cosh \tilde{y}$, and, using the boundary condition, the Usadel equation of the $\mathrm{S}$ layer can be rewritten as

$$
\left(\omega+\frac{\alpha}{\beta}-\frac{\alpha}{\sqrt{2 i} \beta^{2} \tanh \tilde{y}} \cos \theta_{S}\right) \sin \theta_{S}=\Delta \cos \theta_{S} .
$$

Equation (13) yields $\delta \theta_{S}=-\alpha /\left(\beta \Delta_{0}\right)$, and thus no gap in the density-of-states, $\nu(0)=\nu_{0} \alpha /\left(\beta \Delta_{0}\right)$, while 


$$
\delta \Delta=-\frac{\alpha}{\beta}+\frac{\pi \alpha}{8 \beta^{2}} \mathfrak{R}[(1-i) \operatorname{coth} \tilde{y}] .
$$

The screening length is given as

$$
\begin{aligned}
\frac{\lambda_{0}^{2}}{\lambda_{\mathrm{eff}}^{2}(0)}= & 1-\left(1+\frac{2}{\pi}\right) \frac{\alpha}{\beta \Delta_{0}}+\left(\frac{\pi}{8}+\frac{2}{3 \pi}\right) \frac{\alpha}{\beta^{2} \Delta_{0}} \mathfrak{R}[(1 \\
& -i) \operatorname{coth} \tilde{y}]-\frac{\gamma}{16 \beta^{2}} \mathfrak{R}\left[\frac{4 i y+(1+i) \sinh 2 \tilde{y}}{\sinh ^{2} \tilde{y}}\right] .
\end{aligned}
$$

Again the effective screening length oscillates on the scale of $\xi_{F}$. However, these oscillations are suppressed due to the large barrier. For $y \gg 1$, the oscillatory function in the contribution of the $\mathrm{S}$ layer has the same form as the one in Eq. (10) whereas the oscillating part of the contribution from the $\mathrm{F}$ layer is proportional to $y e^{-2 y} \sin (2 y)$.

In the case of a very thin $\mathrm{F}$ layer, $y \ll \beta^{-1}$, the variation of the angle $\theta$ is small across the $\mathrm{F}$ layer (see above). The boundary condition at the SF interface then simplifies, and the Usadel equation of the $\mathrm{S}$ layer yields

$$
\left(\omega+2 i \alpha y+4 \alpha \beta y^{2} \cos \theta_{S}\right) \sin \theta_{S}=\Delta \cos \theta_{S} .
$$

We find $\delta \theta_{S}=-2 i \alpha y / \Delta_{0}$ and $\delta \Delta=-\pi \alpha \beta y^{2}$. As for the case without a barrier, the density of states in the thin-film regime is gapped. Using Eq. (16) to convert the integral over $\omega$ to an integral over $\theta_{S}$, the screening length is given by

$$
\frac{\lambda_{0}^{2}}{\lambda_{\text {eff }}^{2}(0)}=1-\pi\left(1+\frac{16}{3 \pi^{2}}\right) \frac{\alpha \beta}{\Delta_{0}} y^{2}+\gamma y .
$$

The inverse screening length increases in the very narrow regime $d_{F}<\xi_{F}^{3} /\left(\beta \xi_{S}^{2}\right)$.

Thus, we find oscillations of the screening length both in the absence of a barrier and in the presence of a strong barrier. The amplitude of oscillations in the latter case is suppressed, however. In both cases, analytic results can be found for small-thicknesses $y<y^{*}$ and large-thicknesses $y>y^{*}$, where $y^{*} \sim \min \left\{1, \beta^{-1}\right\}$ denotes the position of the first strong minimum. The vicinity of this minimum is not accessible to analytic solution.

To find a solution in this regime, we note that Eq. (2) yields a general relation between $\theta_{0}$ and $\theta_{F}$, namely,

$$
\sin \frac{\theta_{0}}{2}=\frac{1}{\sqrt{1+\cot ^{2} \frac{\theta_{F}}{2} \operatorname{cn}^{2}\left(\tilde{y}, \cos ^{2} \frac{\theta_{F}}{2}\right)}},
$$

where $\mathrm{cn}$ is the Jacobi elliptic function. Using Eq. (18), the boundary condition (3) yields $\theta_{S}$ as a function of $\theta_{F}$. Inserting this solution into the other equations, the set of coupled Eqs. (4) and (5) can then be solved numerically. The thickness dependence of the low-temperature screening length is displayed in Fig. 1. The minimum at $d_{F} \sim \xi_{F}$ is clearly visible whereas further oscillations at larger $d_{F}$ are very small.

Furthermore, the numeric solution allows one to describe the temperature dependence of the screening length. Figure 2 shows the finite temperature curves for different values of $d_{F} / \xi_{F}$. The oscillations of the zero-temperature screening

$$
\frac{\lambda_{0}{ }^{2}}{\lambda_{\mathrm{eff}}^{2}\left(d_{F}\right)}
$$

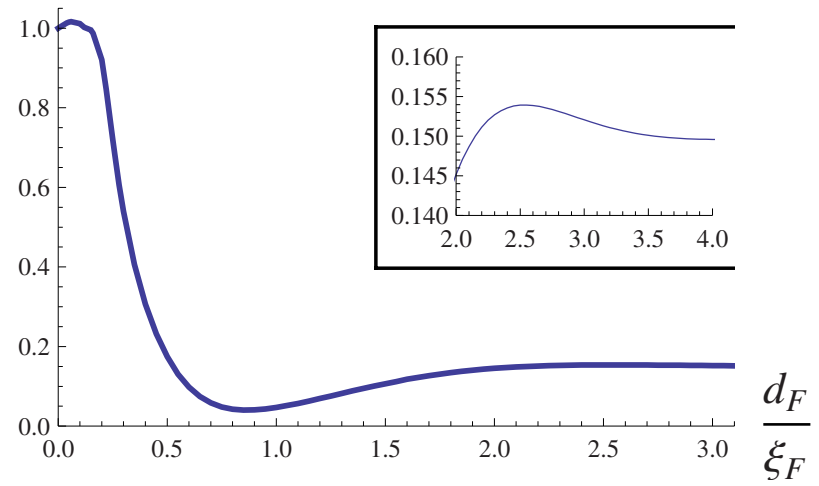

FIG. 1. (Color online) Oscillation of the inverse screeninglength $1 / \lambda_{\text {eff }}^{2}$ as a function of $d_{F}$ at temperature $T=0.1 T_{c 0}$. Here we use the parameters $\alpha=1.2, \beta=1$, and $\gamma=0.6$. The inset magnifies the weak maximum at $d_{F} \approx 2.5 \xi_{F}$.

length mirror the oscillations of the critical temperature as well as the slope of the screening length close to $T_{c}$.

In the vicinity of the critical-temperature $T_{c}$ an analytic solution is possible for all parameter values. For the SF bilayer, the critical temperature is given by the solution of the equation

$$
\ln \frac{T_{c}}{T_{c 0}}=\Psi\left(\frac{1}{2}\right)-\mathfrak{R}\left[\Psi\left(\frac{1}{2}+\frac{1}{2 \pi T_{c} \tau_{s}}\right)\right],
$$

where the (complex) relaxation-time $\tau_{s}$ reads

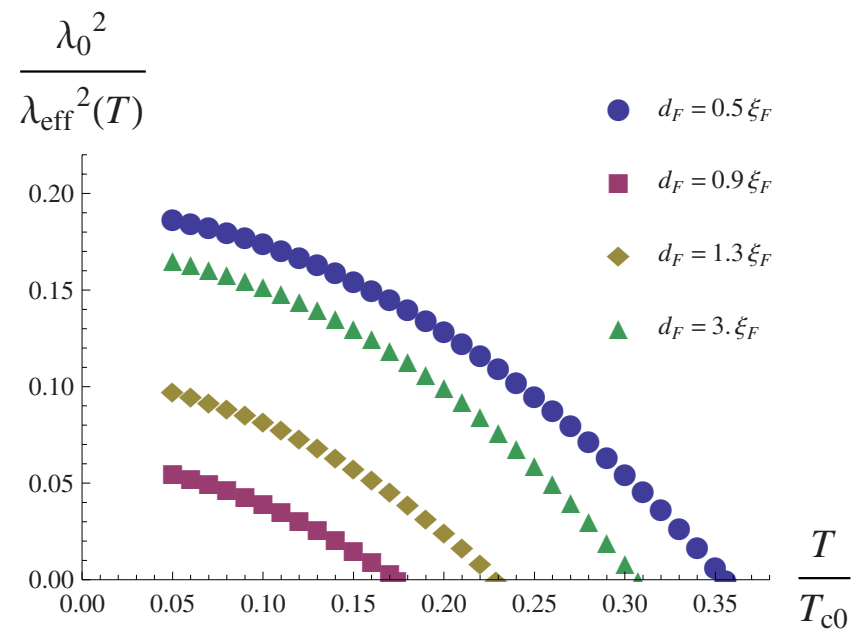

FIG. 2. (Color online) Temperature dependence of $\lambda_{\text {eff }}^{-2}$ The parameters used are the same as in Fig. 1, and four different thicknesses are shown: $d_{F} / \xi_{F}=0.5,0.9,1.3$, and 3 . The corresponding critical temperatures are $T_{c}(0.5)=0.36 T_{c 0}, T_{c}(0.9)=0.17 T_{c 0}$ (close to the minimum), $T_{c}(1.3)=0.23 T_{c 0}$, and $T_{c}(0.5)=0.31 T_{c 0}$ (close to the asymptotic value for $d_{F} / \xi_{F} \gg 1$ ). 


$$
\tau_{s}^{-1}=\frac{\sqrt{2 i} \alpha \tanh (\sqrt{2 i} y)}{1+\sqrt{2 i} \beta \tanh (\sqrt{2 i} y)} .
$$

Equations (19) and (20) are obtained by linearizing (2)-(5) in small $\theta$ close to the transition. For $\tau_{s}^{-1}$ small, $T_{c}=T_{c 0}$ $-\frac{\pi}{4} \mathfrak{R}\left[\tau_{s}^{-1}\right]$. For $\left|\tau_{s}^{-1}\right|=\Delta_{0} / 2$, the transition temperature vanishes according to Eq. (19). Note, however, that for large $\tau_{s}^{-1}$ the transition typically becomes first order. ${ }^{23}$

Expansion of Eqs. (2)-(5) up to cubic order then yields the temperature dependence of the screening-length $\lambda_{\text {eff }}$ close to $T_{c}$. Namely,

$$
\begin{aligned}
\frac{1}{\lambda_{\mathrm{eff}}^{2}(T)}= & \frac{\mu_{0} \sigma_{S}}{\hbar \pi T_{c}} \Delta^{2}(T) \Re\left[\left(1+\frac{\gamma}{4 \sqrt{2 i}} \frac{2 \tilde{y}+\sinh (2 \tilde{y})}{(\cosh \tilde{y}+\tilde{\beta} \sinh \tilde{y})^{2}}\right)\right. \\
& \left.\times \Psi^{(1)}\left(\frac{1}{2}+\frac{1}{2 \pi T_{c} \tau_{s}}\right)\right] .
\end{aligned}
$$

We see that the contribution of the $\mathrm{F}$ layer to $\lambda_{\text {eff }}^{-2}$ displays an oscillatory dependence on its thickness. Furthermore, both $T_{c}$ and $\Delta(T)$ oscillate. In particular,

$$
\frac{\Delta^{2}(T)}{T_{c}-T}=\frac{4 \pi T_{c}\left(1-\mathfrak{R}\left[\left(2 \pi T_{c} \tau_{s}\right)^{-1} \Psi^{(1)}(z)\right]\right)}{-\mathfrak{R}\left[\Psi^{(2)}(z)-f\left(\tau_{s}, \beta, y\right) \Psi^{(3)}(z)\right]},
$$

at $T<T_{c}$, where $z=\frac{1}{2}+\left(2 \pi T_{c} \tau_{s}\right)^{-1}$ and

$$
\begin{aligned}
f\left(\tau_{s}, \beta, y\right)= & \frac{1}{48}\left(2 \pi T_{c} \tau_{s}\right)^{-1} \frac{1}{(1+\widetilde{\beta} \tanh \tilde{y})^{3}} \\
& \times\left(4(1+2 \widetilde{\beta} \tanh \tilde{y})^{2}-\frac{2 \tilde{y}+\sinh (2 \widetilde{y})}{\sinh \tilde{y} \cosh ^{3} \tilde{y}}\right) .
\end{aligned}
$$

Note that the simple-relation $\lambda^{-2} \propto \Delta^{2}$ does not hold in the presence of the $\mathrm{F}$ layer. The slope of $\lambda_{\text {eff }}^{-2}$ close to $T_{c}$ has its own dependence on the thickness of the $\mathrm{F}$ layer and the relaxation-rate $\tau_{s}^{-1}$.

In conclusion, we have shown that the screening length of SF bilayers displays an oscillatory dependence on the thickness of the F layer. Analytic solutions have been found in various regimes and a general solution has been determined numerically. The obtained nonmonotonic dependence of the screening length has been observed experimentally. ${ }^{17}$ Our method can be easily extended to more complicated situations such as multilayers where unusual features of the proximity effect have been predicted. ${ }^{1,2}$

We would like to acknowledge A. Buzdin and T. Lemberger for many discussions. J.S.M. thanks the CEA/INAC/ SPSMS for hospitality.
${ }^{1}$ A. I. Buzdin, Rev. Mod. Phys. 77, 935 (2005).

${ }^{2}$ F. S. Bergeret, A. F. Volkov, and K. B. Efetov, Rev. Mod. Phys. 77, 1321 (2005).

${ }^{3}$ E. A. Demler, G. B. Arnold, and M. R. Beasley, Phys. Rev. B 55, 15174 (1997).

${ }^{4}$ A. I. Buzdin and M. Yu. Kupriyanov, JETP Lett. 52, 487 (1990).

${ }^{5}$ Z. Radovic, M. Ledvij, L. Dobrosavljevic-Grujic, A. I. Buzdin, and J. R. Clem, Phys. Rev. B 44, 759 (1991).

${ }^{6}$ J. S. Jiang, D. Davidovic, D. H. Reich, and C. L. Chien, Phys. Rev. Lett. 74, 314 (1995).

${ }^{7}$ A. I. Buzdin, Phys. Rev. B 62, 11377 (2000).

${ }^{8}$ T. Kontos, M. Aprili, J. Lesueur, and X. Grison, Phys. Rev. Lett. 86, 304 (2001).

${ }^{9}$ A. I. Buzdin, L. N. Bulaevskii, and S. V. Panyukov, JETP Lett. 35, 178 (1982).

${ }^{10}$ A. I. Buzdin and M. Yu. Kupriyanov, JETP Lett. 53, 321 (1991).

${ }^{11}$ V. V. Ryazanov, V. A. Oboznov, A. Yu. Rusanov, A. V. Veretennikov, A. A. Golubov, and J. Aarts, Phys. Rev. Lett. 86, 2427 (2001).

12 A. D. Zaikin, Solid State Commun. 41, 533 (1982).
${ }^{13}$ P. Visani, A. C. Mota, and A. Pollini, Phys. Rev. Lett. 65, 1514 (1990).

${ }^{14}$ F. B. Müller-Allinger and A. C. Mota, Phys. Rev. Lett. 84, 3161 (2000).

${ }^{15}$ S. J. Turneaure, E. R. Ulm, and T. R. Lemberger, J. Appl. Phys. 79, 4221 (1996).

${ }^{16}$ S. J. Turneaure, A. Pesetski, and T. R. Lemberger, J. Appl. Phys. 83, 4334 (1998).

${ }^{17}$ T. R. Lemberger, I. Hetel, A. J. Hauser, and F. Y. Yang, J. Appl. Phys. 103, 07 C701 (2008).

${ }^{18}$ K. D. Usadel, Phys. Rev. Lett. 25, 507 (1970).

${ }^{19}$ M. Y. Kupriyanov and V. F. Lukichev, Sov. Phys. JETP 67, 1163 (1988).

${ }^{20}$ See, e.g., P. G. De Gennes, Superconductivity Of Metals And Alloys (Addison Wesley, Reading, MA, 1989).

${ }^{21}$ I. Baladié and A. I. Buzdin, Phys. Rev. B 67, 014523 (2003).

${ }^{22}$ The numerical values of the coefficients are $a_{1}=1.77, a_{2}=3.82$, $a_{3}=6.44$, and $a_{4}=3.19$.

${ }^{23}$ S. Tollis, Phys. Rev. B 69, 104532 (2004). 